\title{
Gallium Ga 68-labeled-NODAGA-MJ9
}

National Cancer Institute

\section{Source}

National Cancer Institute. Gallium Ga 68-labeled-NODAGA-M19. NCI Thesaurus. Code C115960.

A radiopharmaceutical agent comprised of a peptide targeting the gastrin releasing peptide receptor (GRPR) chelated with 1,4,7-triazacyclononane,1-glutaric acid-4,7 acetic acid (NODAGA) and radiolabeled with gallium Ga 68, with potential tumor imag ing activity upon positron emission tomography (PET). After administration of gallium Ga 68-labeledNODAGA-MJ9, the MJ9 moiety binds to GRPR located on tumor cells. Upon PET imag ing, GRPR-expressing tumor cells can be visualized and expression levels can be quantified. GRPR is overexpressed on certain tumor cells and plays a key role in tumor proliferation and survival. 